\title{
CRESCIMENTO DE CRISTAIS DE RUBI E SAFIRA PELO MÉTODO DO FLUXO
}

\author{
Gislene da Silva Ribeiro \\ Departamento de Recursos Minerais, Universidade Federal de Mato Grosso, Av. Fernando Corrêa da Costa, s/n, \\ 78060-900 Cuiabá - MT, Brasil \\ Rogério Junqueira Prado* \\ Instituto de Física, Universidade Federal de Mato Grosso, Av. Fernando Corrêa da Costa, s/n, 78060-900 Cuiabá - MT, Brasil
}

Recebido em 26/8/09; aceito em 29/1/10; publicado na web em 3/5/10

\begin{abstract}
GROWTH OF RUBY AND SAPPHIRE CRYSTALS BY THE FLUX METHOD. This work reports the growth of corundum crystals by the flux method. The main objective was the evaluation of versatility, effectiveness and real possibilities of the flux method to the synthesis and doping of monocrystals with impurities of particular interest. In this work the chosen impurities were i) $\mathrm{Cr}$ and ii) $\mathrm{Fe}$ and $\mathrm{Ti}$, aiming the synthesis of rubies and sapphires, respectively. The crystals were grown by heating a mixture of $\mathrm{Al}_{2} \mathrm{O}_{3}: \mathrm{Cr}_{\mathrm{Cr}} \mathrm{Al}_{2} \mathrm{O}_{3}: \mathrm{Fe}: \mathrm{Ti}$ and flux $\left(\mathrm{MoO}_{3}\right)$. The maximum crystal size obtained was $1.0 \mathrm{~mm}$, all transparent, presenting well developed faces, bipiramidal hexagonal shape, and showing a typical red (ruby) and/or light blue (sapphire) color. EDX and XPD experiments were performed in order to characterize some of the synthesized crystals. All crystallized specimens presented the $\alpha$-alumina atomic structure.
\end{abstract}

Keywords: flux method; ruby; sapphire.

\section{INTRODUÇÃO}

As gemas, por sua própria natureza, expressam simbolicamente valores e significados dentro do repertório de costumes da vida urbana, sendo conhecidas desde os tempos do Império Romano. ${ }^{1}$ Dentre todas as suas características, a cor pode ser considerada como uma das mais importantes, ${ }^{2}$ pois desempenha um papel fundamental sobre sua beleza, valor e popularidade. ${ }^{3}$ Por exemplo, é justamente devido às suas cores vivas e peculiares que gemas como rubis e safiras (coríndon, $\mathrm{Al}_{2} \mathrm{O}_{3}$ ) estão entre as mais conhecidas e admiradas do mundo.

A origem da cor em minerais está ligada a várias razões, tais como a presença de íons metálicos, fenômenos de transferência de carga, efeitos de radiação ionizante, entre outras. ${ }^{4-6}$

Assim como o coríndon (rubi e safira), berilo (esmeralda, heliodoro, morganita), turmalina, diamante e tantas outras, a maioria das gemas pertence ao reino mineral e, embora não tão abundantes na natureza, são economicamente exploradas por empresas da área de mineração e garimpeiros, mas também podem ser conseguidas através de processos artificiais, o que possui enorme utilidade tanto do ponto de vista comercial quanto do ponto de vista acadêmico e tecnológico. ${ }^{7}$

Foram os mineralogistas franceses do século XIX que sistematizaram e estabeleceram, pela primeira vez, os procedimentos de síntese para a maioria das variedades de gemas, se interessando de maneira muito especial pela cristalização de coríndon. ${ }^{8}$ Safiras e rubis sintéticos têm sido produzidos, comercialmente, desde 1902 pelo processo Verneuil e, posteriormente, por processos hidrotermais e de fluxo. Em 1960 um rubi sintético de Verneuil foi utilizado na fabricação do primeiro laser. ${ }^{8} \mathrm{O}$ laser de rubi tornou-se muito importante para numerosas aplicações, tais como remoção de tatuagens, ${ }^{9,10}$ confecção de fibras sensoras de temperatura ${ }^{11,12}$ e na medicina. ${ }^{13}$

Esses materiais possuem ainda várias aplicações industriais, comerciais e/ou tecnológicas. Rubis e safiras podem ser utilizados na decoração de esferográficas, óculos, relógios e outros objetos sofisticados, na confecção de equipamentos elétricos e óticos bem como em janelas de fornalhas de alta temperatura. Além disso, a safira é um dos poucos materiais isolantes com capacidade de suportar baixíssimas

*e-mail: rjprado@ufmt.br temperaturas ( $4 \mathrm{~K}$, a temperatura do He líquido, por exemplo), sendo por isso muito utilizada em laboratórios de pesquisa. Além do que, safiras com impurezas de titânio (Ti) são usadas em lasers de impulsos ultracurtos. Portanto, a produção de coríndon em escala comercial alcança um grande número de aplicações, motivo pelo qual o domínio de seus processos de síntese é interessante.

Apesar de sua grande importância tecnológica e industrial, a bibliografia relativa à cristalização de pedras preciosas ainda não é suficientemente detalhada, já que as empresas dedicadas a essa atividade guardam sigilosamente os detalhes relativos aos métodos empregados. ${ }^{7}$ São poucos os livros totalmente dedicados à documentação da história e processos de produção de gemas sintéticas, ${ }^{14-16} \mathrm{e}$ há alguns outros que abordam o tema como um tópico ou capítulo específico em seu volume. ${ }^{17-19}$ Nos últimos anos, graças à iniciativa de pesquisadores de universidades e centros de pesquisa, ${ }^{13-16,20-24}$ diversos processos de síntese de gemas têm sido cientificamente desenvolvidos e/ou documentados, e várias técnicas empregadas para o crescimento de gemas sintéticas têm sido descritas, como os métodos de fusão na chama (processo ou modificação de Verneuil), Czochralski, hidrotermal e o método do fluxo, além do processo vapor-líquido-sólido..$^{5,14-16,19}$

Dentre todos, os métodos hidrotermal e do fluxo são, desde seu desenvolvimento, os de mais fácil utilização. Particularmente, o método do fluxo permite o crescimento de cristais em temperatura abaixo do seu ponto de fusão, exigindo estados de supersaturação que podem ser obtidos com relativa facilidade por um processo lento de resfriamento, fluxo de evaporação, ou técnica temperatura-gradiente..$^{5,14-16}$

O principal objetivo deste trabalho foi o de avaliar a versatilidade, eficiência e reais possibilidades do método do fluxo com respeito à síntese de monocristais e dopagem desses cristais com elementos cromóforos e/ou impurezas de interesse. Para isso, foram sintetizados monocristais de coríndon dopados com cromo (rubis) e com ferro e titânio (safiras). Alguns dos monocristais obtidos tiveram suas características morfológicas, químicas e de estrutura cristalina verificadas por microscopia óptica, espectrometria de raios X dispersiva em energia (EDX, Energy Dispersive $X$-ray Spectrometry ${ }^{25}$ ) e difração de raios X (XPD, X-ray Powder Diffraction $^{26}$ ). 


\section{PARTE EXPERIMENTAL}

O método de fluxo ${ }^{14-16,20,24}$ foi utilizado neste trabalho como o ponto de partida para o crescimento de monocristais de coríndon (rubis e safiras) e consiste na dissolução do material que se pretende cristalizar (soluto) em um composto fundido (fluxo), que atua como solvente e se evapora durante o processo em alta temperatura, aumentando a concentração de soluto na solução, que então se precipita e cristaliza.

Como é bem conhecido da literatura, a cor vermelha do rubi natural é devida à existência de íons $\mathrm{Cr}^{+3}$ na estrutura da alumina, enquanto que a cor da safira azul está relacionada à presença de pares de ferro $\left(\mathrm{Fe}^{+2}\right)$ e titânio $\left(\mathrm{Ti}^{+4}\right)$ em sua estrutura cristalina. ${ }^{4}$ Neste último caso, é a transferência de elétrons do Fe para o Ti vizinho, que envolve a absorção da luz amarela, e acaba tendo por resultado a transmissão da cor azul complementar pelo cristal. ${ }^{5}$ Se apenas átomos isolados de Ti ou Fe estão presentes na safira esta cor não é obtida. Temos, portanto, que rubis e safiras possuem características químicas e estruturais semelhantes, variando-se apenas os componentes (ou impurezas) responsáveis pela cor característica do mineral. ${ }^{6}$

Assim sendo, cristais de rubi foram crescidos utilizando $\mathrm{MoO}_{3}$ (Sigma-Aldrich, 99,5\%) como fluxo, enquanto que uma mistura de reagente grau analítico $\mathrm{Al}_{2} \mathrm{O}_{3}$ e óxido dopante $\mathrm{Cr}_{2} \mathrm{O}_{3}$ foi usada como soluto, segundo a reação apresentada a seguir:

$$
\mathrm{x} \mathrm{Al}_{2} \mathrm{O}_{3}+(1-\mathrm{x}) \mathrm{Cr}_{2} \mathrm{O}_{3}+3 \mathrm{MoO}_{3} \rightarrow \mathrm{Al}_{2 \mathrm{x}} \mathrm{Cr}_{2-2 \mathrm{x}} \mathrm{Mo}_{3} \mathrm{O}_{12}
$$

Óxido de cromo $\left(\mathrm{Cr}_{2} \mathrm{O}_{3} / 0,0050 \mathrm{~g} / 0,5 \%\right.$ em massa do soluto), óxido de alumínio $\left(\mathrm{Al}_{2} \mathrm{O}_{3} / 1,0050 \mathrm{~g}\right)$ e trióxido de molibdênio $\left(\mathrm{MoO}_{3} / 19,000\right.$ g), todos na forma de pó, foram pesados e depois misturados num almofariz de ágata. A mistura foi colocada num cadinho de platina com tampa, de $30 \mathrm{~cm}^{3}$ de capacidade, e levada a um forno mufla (marca Analítica, modelo AN1406) com elementos aquecedores de carbeto de silício ( $\mathrm{SiC}$ ), uma vez que resistências comuns são atacadas pelo $\mathrm{MoO}_{3}$ que se evapora do cadinho. $\mathrm{O}$ forno foi aquecido a uma taxa de $45^{\circ} \mathrm{C} \mathrm{h}^{-1}$ até a temperatura de $1100{ }^{\circ} \mathrm{C}$, a qual foi mantida durante 5 $\mathrm{h}$. A cristalização se deu por resfriamento, a uma taxa que variou de 30 a $150{ }^{\circ} \mathrm{C} \mathrm{h}^{-1}$, dependendo da amostra sintetizada.

$\mathrm{O}$ mesmo processo foi também usado para a síntese das safiras. Apenas os íons cromóforos, neste caso $\mathrm{TiO}_{2}$ e $\mathrm{Fe}_{2} \mathrm{O}_{3}$ (Merck, P.A.), foram adicionados em igual quantidade, de tal forma que a concentração total de íons cromóforos fosse igual a $0,5 \%$ em massa do $\mathrm{Al}_{2} \mathrm{O}_{3}$ presente na mistura.

Os cristais sintetizados foram inicialmente analisados por microscopia óptica. A técnica de XPD foi utilizada para a identificação da estrutura cristalina das amostras sintetizadas, enquanto que a técnica de EDX foi utilizada para a caracterização química das mesmas. Para a realização desses estudos as amostras foram pulverizadas utilizandose almofariz e pistilo de ágata até alcançarem granulometria adequada, sendo então analisadas na forma de pó por XPD e EDX.

As análises de difração de raios $\mathrm{X}$ foram realizadas num difratômetro modelo XRD-6000 (Shimadzu) equipado com tubo de $\mathrm{Cu}$ (operando a 1,2 kW) e monocromador de grafite. As varreduras foram realizadas em geometria $\theta-2 \theta$ (Bragg-Brentano), entre $10^{\circ}$ e $100^{\circ}$

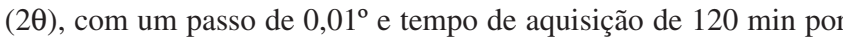
difratograma. Antes da aquisição do difratograma de cada amostra de coríndon analisada, foi realizado o alinhamento óptico do difratômetro, seguido da medida de uma amostra de Si padrão (Shimadzu, 300 mesh), que sempre forneceu diferenças menores que $0,005^{\circ}$ entre as posições esperadas e verificadas para os picos de difração do $\mathrm{Si}$, o que indica o alto grau de precisão do equipamento e o ótimo alinhamento óptico realizado. Todavia, devido à pequena quantidade de material disponível, ao invés de se utilizar um porta-amostra de carga frontal, as amostras foram depositadas sobre um substrato de vidro preparado com uma fina camada de graxa de silicone. Para isso utilizou-se uma peneira de 0,062 $\mathrm{mm}$. Este procedimento foi necessário para reduzir os efeitos de orientação preferencial observados para as amostras de coríndon analisadas com porta-amostra de carga frontal.

A análise química das amostras foi realizada pela técnica de EDX utilizando um espectrômetro modelo EDX-700HS (Shimadzu) equipado com tubo de Rh e feixe de $1 \mathrm{~mm}$ de diâmetro. Foram empregados dois canais analíticos durante o processo, o primeiro para a quantificação de elementos químicos do $\mathrm{Na}$ ao $\mathrm{Sc}$, com tensão no tubo de $15 \mathrm{kV}$, e o segundo para a quantificação de elementos do Ti ao U, com tensão no tubo de $50 \mathrm{kV}$. As medidas foram realizadas em vácuo com tempo de aquisição de $200 \mathrm{~s}$ por canal analítico. Os espectros foram interpretados através do programa $E D X$-Software da Shimadzu $^{\circledR}$, utilizando o método Qual-Quant FP, um método analítico por parâmetros fundamentais que se utiliza de padrões internos. Amostras na forma de pó, com quantidades conhecidas de $\mathrm{Al}_{2} \mathrm{O}_{3}$ e impurezas foram utilizadas para verificar a precisão do método. As incertezas estimadas foram inferiores a 0,5 e $0,1 \%$, para o $\mathrm{Al}_{2} \mathrm{O}_{3}$ e dopantes, respectivamente. Elementos com números atômicos menores que 11 (Na) não podem ser detectados e quantificados por este equipamento.

\section{RESULTADOS E DISCUSSÃO}

Fotos típicas dos cristais de rubi e safira sintetizados são mostradas nas Figuras 1 e 2, respectivamente. Foram obtidos cristais de rubi e safira transparentes, com tamanho máximo de $1 \mathrm{~mm}$, e que apresentaram coloração vermelha clara (rubis) e azul claro (safiras), faces bem desenvolvidas e forma hexagonal bipiramidal, que segundo a literatura é atribuída a uma maior adsorção de cadeias epitaxiais de $\mathrm{MoO}_{3}$ pelas faces $\{11 \overline{2} 3\}$ dos cristais, ${ }^{13}$ também ilustradas na Figura 2.

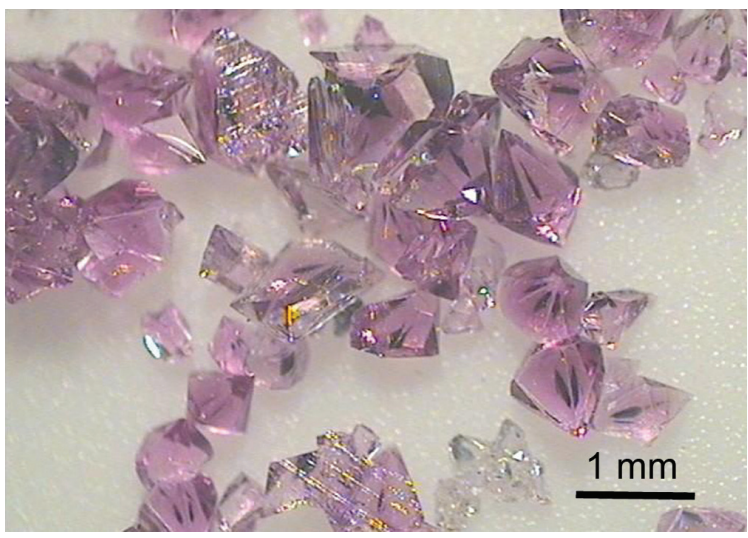

Figura 1. Micrografia óptica de vários dos rubis sintetizados
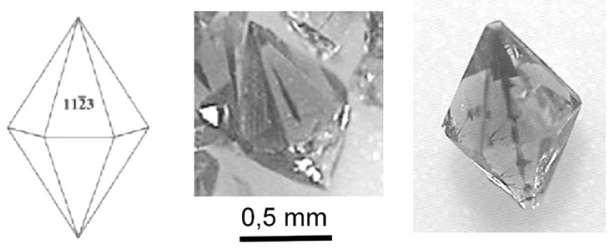

Figura 2. Desenho de um cristal com forma de bipirâmide hexagonal, e cristais de rubi e safira sintetizados

De maneira geral, os cristais obtidos com taxas de resfriamento mais altas $\left(150^{\circ} \mathrm{C} \mathrm{h}^{-1}\right)$ foram bem maiores que os obtidos com resfriamentos mais demorados $\left(30^{\circ} \mathrm{C} \mathrm{h}^{-1}\right)$, que em geral produziram um maior número de cristais, mas cristais de menor tamanho.

Segundo os dados cristalográficos aceitos para o cristal, o arranjo atômico do coríndon pertence a um grupo espacial hexagonal, cujos 
parâmetros de cela unitária e posições atômicas da literatura ${ }^{27,28}$ são citados na Tabela 1, enquanto que a representação gráfica da cela unitária e arranjo atômico correspondente estão ilustrados na Figura 3. Este arranjo hexagonal é formado por camadas de oxigênio, entre as quais se encontram cátions com coordenação octaédrica, cada um ligado a seis íons de oxigênio. Todavia, apenas cerca de dois terços das posições disponíveis para os cátions estão em geral ocupadas. Cada face comum a dois octaedros vizinhos é formada por grupos de três íons oxigênio, cada um deles ligado a um par de íons Al, localizados no centro de cada octaedro mostrado na Figura 3.

Tabela 1. Dados estruturais para o coríndon $\left(\mathrm{Al}_{2} \mathrm{O}_{3}\right)$. Adaptado da ref. 7

\begin{tabular}{lcc}
\hline $\mathrm{Al}_{2} \mathrm{O}_{3}$ & & \\
\hline \multirow{3}{*}{ Cela unitária } & $\mathrm{a}(\AA)$ & 4,754 \\
& $\mathrm{c}(\AA)$ & 12,99 \\
\multirow{2}{*}{ Grupo espacial } & $\mathrm{V}\left(\AA^{3}\right)$ & 254,25 \\
\multirow{2}{*}{ Posições atômicas } & $\rho\left(\mathrm{g} / \mathrm{cm}^{3}\right)$ & 3,99 \\
& \multicolumn{2}{c}{$\mathrm{P} 6(3) / \mathrm{mmc}$} \\
\hline
\end{tabular}

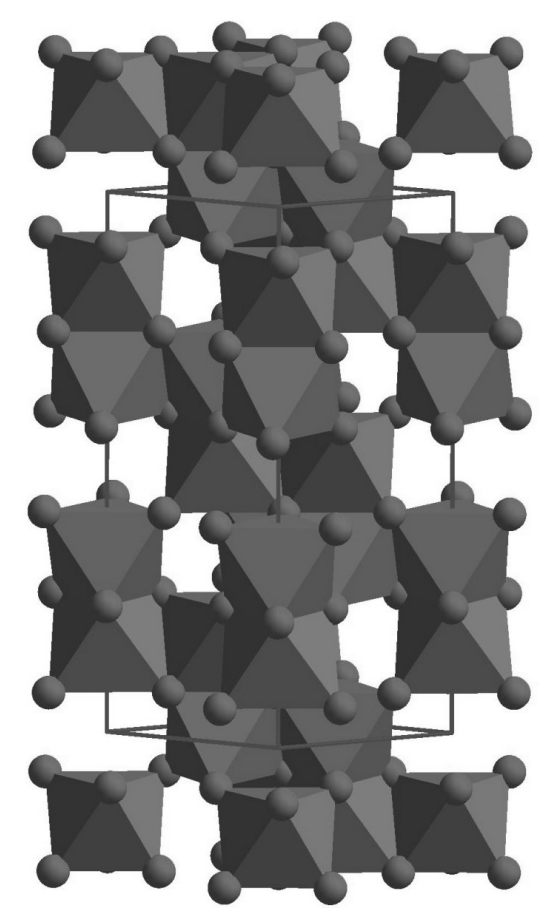

Figura 3. Estrutura atômica do coríndon. Adaptado da ref. 7

Todos os difratogramas obtidos para os cristais sintetizados foram compatíveis com o perfil esperado para a estrutura cristalina da $\alpha$-alumina, disponível na base de dados do ICSD (Inorganic Crystal Structure Database), cartões número \#10425 ou \#92629. ${ }^{27,28}$ Portanto, se existem outras fases além da própria alumina nas amostras, suas proporções, com relação à fase majoritária, estão abaixo da sensibilidade da técnica de XPD. Os difratogramas obtidos atestam o alto grau de cristalinidade das amostras sintetizadas, através do pequeno valor obtido para a largura a meia altura, da ordem de 0,10 a $0,14^{\circ} \mathrm{em} 2 \theta$, exatamente na mesma faixa de valores obtidos para a largura a meia altura dos picos de difração de monocristais de silício cristalino e quartzo cristalino pulverizados com gral de ágata, quando utilizadas as mesmas condições de medida experimentais. A largura a meia altura de alguns dos picos de difração das safiras e rubis sintetizados era, inclusive, menor que a de picos dos cristais de referência para a mesma faixa angular.
A Figura 4 mostra o difratograma de uma das amostras de rubi analisadas, juntamente com o difratograma teórico esperado para a estrutura número \#10425 do ICSD. ${ }^{27}$

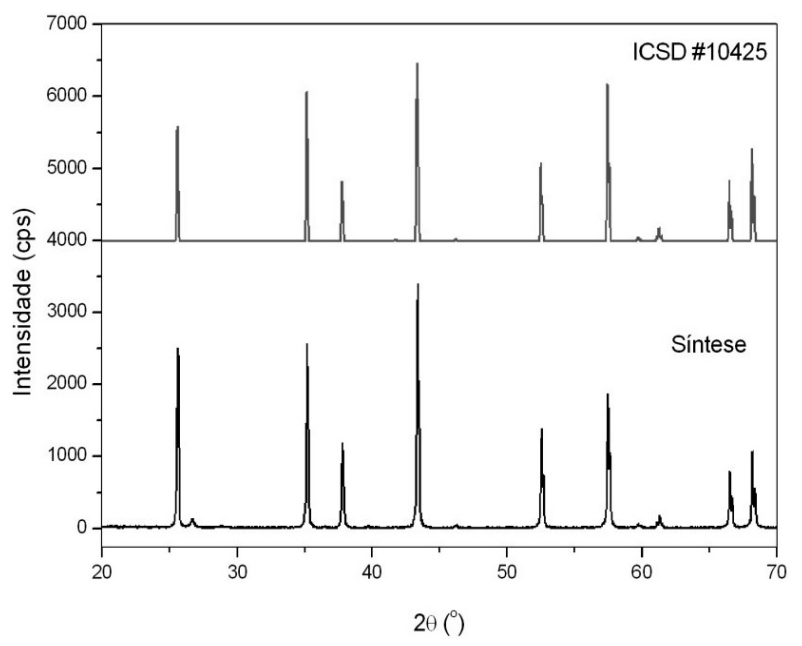

Figura 4. Difratograma obtido para uma das amostras de rubi sintetizadas pelo método do fluxo e sua comparação com dados da literatura. Adaptado da ref. 7

As intensidades normalizadas de alguns dos planos cristalinos, juntamente com seus respectivos índices de Miller, e as distâncias interplanares obtidas para os rubis e as safiras sintetizados são dadas nas Tabelas 2 e 3, respectivamente.

Tabela 2. Espaçamento interplanar, índice de Miller e intensidade relativa dos picos de difração do rubi $\left(\mathrm{Al}_{2} \mathrm{O}_{3}: \mathrm{Cr}\right)$, juntamente com dados comparativos da literatura (ICSD, estrutura \#10425)

\begin{tabular}{lcccccc}
\hline $2 \theta /^{\circ}$ & $2 \theta_{\mathrm{lit}}{ }^{\mathrm{o}}$ & $\mathrm{d} / \mathrm{nm}$ & $\mathrm{d}_{\mathrm{lit}} / \mathrm{nm}$ & $(h k l)$ & $I / I_{0}$ & $\left(\mathrm{I} / \mathrm{I}_{0}\right)_{\text {lit. }}$ \\
\hline 25.618 & 25.596 & 0.34745 & 0.34773 & $10-2$ & 64 & 58.6 \\
35.181 & 35.167 & 0.25488 & 0.25498 & 104 & 70 & 95.2 \\
37.805 & 37.817 & 0.23778 & 0.2377 & 110 & 32 & 44.5 \\
43.389 & 43.391 & 0.20838 & 0.20837 & 113 & 100 & 96.1 \\
52.593 & 52.595 & 0.17388 & 0.17387 & $20-4$ & 41 & 50.1 \\
57.526 & 57.533 & 0.16008 & 0.16006 & 116 & 59 & 100 \\
66.541 & 66.583 & 0.14041 & 0.14033 & 214 & 25 & 40.8 \\
68.229 & 68.289 & 0.13735 & 0.13724 & 300 & 33 & 62.8 \\
\hline
\end{tabular}

Tabela 3. Espaçamento interplanar, índice de Miller e intensidade relativa dos picos de difração da safira $\left(\mathrm{Al}_{2} \mathrm{O}_{3}: \mathrm{Fe}: \mathrm{Ti}\right)$ juntamente com dados comparativos da literatura (ICSD, estrutura \#10425)

\begin{tabular}{lcccccc}
\hline $2 \theta /{ }^{\circ}$ & $2 \theta_{\mathrm{lit}}{ }^{\circ}$ & $\mathrm{d} / \mathrm{nm}$ & $\mathrm{d}_{\mathrm{lit}} / \mathrm{nm}$ & $(h k l)$ & $I / I_{0}$ & $\left(\mathrm{I} / \mathrm{I}_{0}\right)_{\text {lit. }}$ \\
\hline 25.634 & 25.596 & 0.34723 & 0.34773 & $10-2$ & 37 & 58.6 \\
35.214 & 35.167 & 0.25466 & 0.25498 & 104 & 56 & 95.2 \\
37.833 & 37.817 & 0.23761 & 0.2377 & 110 & 24 & 44.5 \\
43.414 & 43.391 & 0.20827 & 0.20837 & 113 & 100 & 96.1 \\
52.605 & 52.595 & 0.17384 & 0.17387 & $20-4$ & 35 & 50.1 \\
57.566 & 57.533 & 0.15998 & 0.16006 & 116 & 73 & 100 \\
66.570 & 66.583 & 0.14036 & 0.14033 & 214 & 28 & 40.8 \\
68.254 & 68.289 & 0.13730 & 0.13724 & 300 & 30 & 62.8 \\
\hline
\end{tabular}

Os dados de XPD obtidos, por si só, atestam a obtenção de rubis e safiras sintéticas. Esta informação é também corroborada pelo formato bipiramidal hexagonal dos cristais e pela análise de EDX dos mesmos, o que não deixa dúvidas a esse respeito.

Além do alumínio, outros elementos químicos também foram identificados e quantificados por EDX, mas estão presentes em 
pequena quantidade. A Tabela 4 exibe os dados de EDX (em porcentagem de massa, wt.\%) obtidos para as amostras sintetizadas. Observa-se que as amostras de rubi e safira ( $\alpha$-alumina) analisadas apresentaram ótimos índices de pureza, todos superiores a $99 \mathrm{wt} . \%$ (para o conteúdo de $\mathrm{Al}_{2} \mathrm{O}_{3}+\mathrm{Cr}_{2} \mathrm{O}_{3}$ ), atestando assim o alto grau de qualidade dos compostos utilizados.

Tabela 4. Composição química dos rubis e safiras sintetizadas neste trabalho, obtida por EDX

\begin{tabular}{lcccc}
\hline & $\begin{array}{c}\text { Rubi1 } \\
\text { wt.\% }\end{array}$ & $\begin{array}{c}\text { Rubi2 } \\
\text { wt.\% }\end{array}$ & $\begin{array}{c}\text { Rubi3 } \\
\text { wt.\% }\end{array}$ & $\begin{array}{c}\text { Safira 01 } \\
\text { wt\% }\end{array}$ \\
\hline $\mathrm{Al}_{2} \mathrm{O}_{3}$ & 99.16 & 99.00 & 98.84 & 99.92 \\
$\mathrm{Cr}_{2} \mathrm{O}_{3}$ & 0.35 & 0.43 & 0.40 & ---- \\
$\mathrm{TiO}_{2}$ & ---- & 0.01 & ---- & 0.02 \\
$\mathrm{Fe}_{2} \mathrm{O}_{3}$ & 0.03 & 0.04 & 0.06 & 0.02 \\
$\mathrm{MoO}_{3}$ & 0.06 & 0.32 & 0.51 & 0.03 \\
$\mathrm{CaO}_{2}$ & ---- & 0.08 & 0.06 & 0.004 \\
$\mathrm{P}_{2} \mathrm{O}_{5}$ & 0.07 & ---- & ---- & ---- \\
$\mathrm{K}_{2} \mathrm{O}$ & ---- & 0.07 & 0.09 & 0.008 \\
$\mathrm{ZnO}$ & 0.003 & 0.005 & ---- & $<0.001$ \\
$\mathrm{NbO}^{\mathrm{V}_{2} \mathrm{O}_{5}}$ & 0.004 & ---- & ---- & ---- \\
$\mathrm{PtO}_{2}$ & ---- & 0.02 & ---- & ---- \\
$\mathrm{CeO}_{2}$ & 0.03 & ---- & ---- & ---- \\
$\mathrm{WO}_{3}$ & 0.3 & ---- & ---- & ---- \\
$\mathrm{Ga}_{2} \mathrm{O}_{3}$ & ---- & 0.03 & 0.04 & 0.01 \\
\hline
\end{tabular}

Os rubis 2 e 3 apresentaram ainda quantidades razoáveis de molibdênio (entre 0,3 e 0,5 wt.\%). Todavia, as outras duas amostras apresentaram concentrações praticamente desprezíveis, menores que 0,1 wt. \%. Deve ser ressaltado que, devido à necessidade de boa quantidade de amostra em pó para a realização das análises de XPD e EDX, não somente alguns dos maiores cristais formados foram pulverizados, mas também todos os menores. Se apenas os maiores cristais tivessem sido pulverizados, é possível que uma concentração mais uniforme e menor de Mo fosse determinada para cada amostra. Isso porque os maiores cristais aparentam, por microscopia óptica, uma maior pureza e homogeneidade.

Os elementos $\mathrm{Cr}$, Fe e Ti foram incorporados ao soluto de maneira proposital, para que fossem incorporados às amostras sintetizadas com a finalidade de dar cor às mesmas. Processo similar poderia ser facilmente utilizado com outros elementos químicos, com a finalidade de dopar os cristais sintetizados com as impurezas desejadas, de tal maneira a modificar alguma das características físicas do cristal (magnéticas, ópticas etc.). Apesar de colocados em quantidades similares (de 0,5 wt.\% do soluto) a incorporação dos íons $\mathrm{Cr}$ nos cristais de rubi sintetizados $(\sim 0,4$ wt.\%) mostrou-se mais eficiente que a incorporação de Fe e Ti nos cristais de safira ( 0,04 wt.\%), que foi pelo menos uma ordem de grandeza inferior à quantidade disponível dos mesmos no soluto. Não foram encontradas indicações deste fato em trabalhos da literatura, sendo que, em geral, se supôs que as impurezas se incorporassem nos cristais com as mesmas concentrações nas quais estavam presentes no soluto.

Há, aparentemente, uma maior compatibilidade cristalo-química do sítio octaédrico do $\mathrm{Al}^{+3}$ por $\mathrm{Cr}^{+3}$, provavelmente devido não somente ao estado de oxidação idêntico (o que não ocorre com o $\mathrm{Ti}^{+4}$, por exemplo), mas também devido a seus raios iônicos $(1,82$ e 1,85 , para o $\mathrm{Al}$ e $\mathrm{Cr}$, respectivamente). Ferro e titânio têm raios iônicos de 1,72 e $2,00 \AA$, respectivamente, bem diferentes do valor apresentado pelo alumínio. Assim, o cromo possui estado de oxidação e raio iônico praticamente idêntico ao do alumínio, facilitando sua incorporação de maneira substitucional no cristal de coríndon. Por outro lado, o titânio tem raio iônico e estado de oxidação diferentes daquele do Al, o que dificulta sua incorporação durante o crescimento do cristal, a não ser quando este se incorpora vizinho a um átomo de ferro que, por sua vez, possui raio iônico menor que o do Al, facilitando a incorporação de um átomo maior em um octaedro vizinho.

A dificuldade em se substituir o $\mathrm{Al}$ por Fe e Ti através da adição pura e simples de $\mathrm{Fe}_{2} \mathrm{O}_{3}$ e TiO na solução de partida fica evidente, não apenas na baixa concentração destes óxidos nas safiras sintetizadas analisadas por EDX, mas também na fraca coloração obtida para essas mesmas amostras, evidenciando-se essa baixa concentração de elementos cromóforos através da pouca transferência de carga entre átomos de Fe e Ti em octaedros vizinhos.

Átomos de Pt e Mo eram esperados em baixa concentração, uma vez que parte do molibdênio do fluxo pode não evaporar completamente, permanecendo nas amostras sintetizadas, que também podem se contaminar com a platina do cadinho, como experimentalmente verificado por EDX. Como as amostras de rubi e safira foram pulverizadas com almofariz e pistilo de ágata para a realização das análises de EDX e XPD, a contaminação com Si encontrada nas mesmas, decorrente da pulverização, foi desconsiderada durante a análise dos dados de EDX. Esta pequena contaminação pode ser também observada no difratograma, como mostra o pico em $26,68^{\circ} \mathrm{em} 2 \theta$, referente ao plano [101] do quartzo presente na amostra (contaminação proveniente do gral de ágata, durante o processo de pulverização). Com relação a este fato, uma amostra de rubi sintetizada foi triturada e fundida em tetraborato de lítio, e a pérola vítrea proveniente desta análise não apresentou contaminação por silício.

A análise por EDX também detectou outros elementos químicos (impurezas) nos cristais sintetizados, como Ca, K, Zn, Nb, V, W, Ga e Ce, mas em concentrações praticamente desprezíveis $(<0,1 \%)$. Vale ressaltar que as linhas características utilizadas para a identificação e quantificação dessas impurezas não podem ser geradas pelos componentes principais dos sistemas estudados ( $\mathrm{Al}, \mathrm{O}, \mathrm{Fe}$, Ti e $\mathrm{Cr}$ ). O fluxo $\left(\mathrm{MoO}_{3}\right)$ e os precursores utilizados $\left(\mathrm{Al}_{2} \mathrm{O}_{3}, \mathrm{Cr}_{2} \mathrm{O}_{3}, \mathrm{TiO}_{2}\right.$ e Fe $\left.\mathrm{F}_{3}\right)$ foram também analisados por EDX, e não apresentaram traços dos elementos acima citados, comprovando que os materiais de partida não são a fonte de contaminação dos cristais sintetizados. Esta pequena contaminação é, muito provavelmente, proveniente do refratário do forno mufla utilizado, já que há um desprendimento contínuo de pó do mesmo sob altas temperaturas e, apesar de as sínteses terem sido realizadas com a utilização de uma tampa de platina para o cadinho, esta não protegia completamente todo o cadinho, deixando frestas pelas quais a poeira do refratário pode ter contaminado a mistura fundida. Além disso, é possível que o óxido de molibdênio evaporado reaja com o refratário do cadinho, atacando-o quimicamente, fazendo com que a contaminação durante o processo de síntese seja maior que durante um tratamento térmico convencional.

É interessante também que as concentrações de Fe são maiores nas amostras de rubi, nas quais não foi utilizado esse elemento durante a síntese, do que nas amostras de safira. Provavelmente, a presença de $\mathrm{Cr}$ na mistura acaba por facilitar também a incorporação de $\mathrm{Fe}$, presente em baixa concentração no $\mathrm{Cr}_{2} \mathrm{O}_{3}$ utilizado como dopante, ou mesmo como resultado de alguma contaminação, visto que a incorporação de Fe durante a síntese de safiras foi muito menos importante.

\section{CONCLUSÕES}

Cristais de rubi e safira apresentando faces bem desenvolvidas e forma hexagonal bipiramidal foram sintetizados com sucesso através do método do fluxo utilizando trióxido de molibdênio como solvente.

Todas as amostras de rubis analisadas apresentaram concentrações de $\mathrm{Al}_{2} \mathrm{O}_{3}$ de pelo menos 99 wt. $\%$ e de $\mathrm{Cr}_{2} \mathrm{O}_{3}$ entre 0,35 e 0,43 wt. $\%$. 
Nota-se, então, que a quantidade de $\mathrm{Cr}$ foi incorporada na estrutura de uma forma significativa, e em quantidade similar à utilizada no soluto. O mesmo não aconteceu para o Fe e Ti utilizados como dopante nas safiras, que se incorporaram nos cristais em quantidade aproximadamente 10 vezes menor que a utilizada no soluto, indicando que nem todas as impurezas serão incorporadas no cristal sintetizado nas proporções que estão presentes no soluto.

Além da composição característica dos rubis e safiras sintetizados, formada principalmente de $\mathrm{Al}_{2} \mathrm{O}_{3}$, a técnica de EDX possibilitou a identificação e quantificação de impurezas presentes em pequenas quantidades (<0,1\% em massa), como Ca, K, Zn, Nb, V, W, Ga e Ce, provenientes do refratário do forno utilizado no processo de síntese.

Os difratogramas de raios $\mathrm{X}$ obtidos dos cristais de rubi e safira sintetizados atestam o alto grau de cristalinidade obtido, sendo que todos os difratogramas foram identificados como $\alpha-\mathrm{Al}_{2} \mathrm{O}_{3}$ pelo software de busca presente no difratômetro (ICSD, cartões número \#10425 ou \#92629).

Esses resultados mostram que a síntese de cristais pelo método do fluxo é muito eficiente para a produção de monocristais dopados. Além de elementos tipicamente cromóforos, poderiam ser utilizadas impurezas com propriedades magnéticas, por exemplo, com o intuito de se estudar a ordem local ao redor da impureza, bem como a influência dessas impurezas nas características magnéticas do material sintetizado.

Devido à facilidade de implementação do método, mesmo em laboratórios que tenham apenas uma pequena quantidade de recursos disponíveis, e sua versatilidade, que permite a produção de uma grande quantidade de cristais com os mais diferentes constituintes, esse método se mostra ideal para a produção de amostras cristalinas de interesse científico.

\section{AGRADECIMENTOS}

Ao Laboratório Multiusuário de Técnicas Analíticas (LAMUTA/ UFMT), onde as análises e sínteses foram realizadas, e aos órgãos de fomento FINEP, CNPq e FAPEMAT (Fundação de Amparo à Pesquisa de Mato Grosso) que, de diversas maneiras, vêm financiando a implantação e melhoria da infraestrutura de pesquisa da UFMT, o que por sua vez possibilitou o desenvolvimento deste trabalho.

\section{REFERÊNCIAS}

1. Guimarães, F. L. N.; Schnellrath, J.; Série Monográficas do CETEM, falta editora: Rio de Janeiro, 2002.
2. Schumann, W.; Rochas e Minerais, $1^{\mathrm{a}}$ ed., Ao Livro Técnico S/A: Rio de Janeiro, 1982.

3. Anderson, B. W.; A identificação das gemas, Ed. Ao Livro Técnico: Rio de Janeiro, 1993.

4. Hankin, R.; Gemas, Cristais e Minerais, 1a ed., Edições 70: Lisboa, 1998.

5. Jayaraman, A.; Curr. Sci. 2000, 79, 1555.

6. O’Donoghue, M.: Joyner, L.; Identification of Gemstones, 1르. ed., Butterworth-Heinemann: Oxford, 2003.

7. Cornejo, M. V. L. A.; Historia Natural (Espanha) 2004, 5, 54.

8. Ruedlinger, R.; J. Cutaneous Laser Therapy 2000, 2, 37.

9. Kupermanbeade, M.; Levine, V. J.; Ashinoff, R.; Am. J. Clinical Dermatology 2001, 2, 21.

10. Grattan, K. T. V.; Zhang, Z. Y.; Suan, T.; Shen, L.; Tong, L.; Ding, Z.; MSTCEP 2001, 981.

11. Seat, H. C.; Sharp, J. H.; Zhang, Z. Y.; Grattan, K. T. V.; SAAPEB 2002 , $101,24$.

12. Moore, J.; Silva, S. R.; Lasers in Medical Sci. 2009, 24, 137.

13. Oishi, S.; Teshima, K.; Hondo, H.; JACSAT 2004, 126, 4768.

14. Nassau, K. Em Gems Made by Man; Nassau, K., ed.; Chilton Book Company: Radnor, 1980, cap. 7.

15. Ewell, D.; Man-made Gemstones, Ellis Horwood: New York, 1979.

16. O’Donoghue, M.; Artificial Gemstones, Robert Hale \& Company: Londres, 2007.

17. O'Donoghue, M. Em A Guide to Man-made Gemstones; Van Nostrand Reinhold; Olympic Marketing Corp.: New York, 1983.

18. Read, P. G.; Gemmology, Robert Hale \& Company: Londres, 2007. $3^{\text {a }}$ ed., 2009.

19. O'Donoghue, M.; Synthetic, Imitation and Treated Gemstones, Butterworth-Heinemann: Oxford, 1997.

20. Teshima, K.; Matsumoto, K.; Hondo, H.; Suzuki, T.; Oishi, S.; JCSJEW 2007, 115, 379.

21. Oishi, S.; Yamamoto, H.; JMACEP 1996, 6, 1687.

22. Patil, L. A.; Wani, P. A.; CRTEDF 2001, 36, 371.

23. Patil, L. A.; Mahanubhav, M. D.; BUMSDW 2007, 30, 141.

24. Scheel, H. J.; JCRGAE SM 1974, 24/25, 669.

25. Jenkins, R.; Vries, J. L. Em Practical X-ray Spectrometry; Jenkins, R.; Vries, J. L.; Philips Tech. Library: Eindhoven, $2^{\mathrm{a}}$ ed., 1972.

26. Cullity, B. D.; Elements of X-Ray Diffraction, $2^{\text {a }}$ ed., Addison-Wesley: Reading, 1978.

27. Ishizawa, N.; Miyata, T.; ASBSDK 1980, 36, 228.

28. http://www.portaldapesquisa. com.br/databases/sites, acessada em Julho 2009. 